\title{
Adaptive radiotherapy for head and neck cancer reduces the requirement for rescans during treatment due to spinal cord dose
}

\author{
Louise Belshaw ${ }^{1}$, Christina E. Agnew ${ }^{1}$, Denise M. Irvine ${ }^{1}$, Keith P. Rooney ${ }^{2}$ and Conor K. McGarry ${ }^{1,3^{*}}$
}

\begin{abstract}
Background: Patients treated with radiotherapy for head and neck (H\&N) cancer often experience anatomical changes. The potential compromises to Planning Target Volume (PTV) coverage or Organ at Risk (OAR) sparing has prompted the use of adaptive radiotherapy (ART) for these patients. However, implementation of ART is time and resource intensive. This study seeks to define a clinical trigger for H\&N re-plans based on spinal cord safety using $\mathrm{kV}$ Cone-Beam Computed Tomography (CBCT) verification imaging, in order to best balance clinical benefit with additional workload.

Methods: Thirty-one H\&N patients treated with Volumetric Modulated Arc Therapy (VMAT) who had a rescan CT $(\mathrm{rCT})$ during treatment were included in this study. Contour volume changes between the planning $\mathrm{CT}(\mathrm{pCT})$ and $\mathrm{rCT}$ were determined. The original treatment plan was calculated on the $\mathrm{PCT}, \mathrm{CBCT}$ prior to the $\mathrm{rCT}$, $\mathrm{pCT}$ deformed to the anatomy of the CBCT ( $\mathrm{dCT}$ ), and $\mathrm{rCT}$ (considered the gold standard). The dose to $0.1 \mathrm{cc}$ (D0.1 $\mathrm{cc}$ ) spinal cord was evaluated from the Dose Volume Histograms (DVHs).
\end{abstract}

Results: The median dose increase to D0.1cc between the pCT and rCT was 0.7 Gy (inter-quartile range 0.2-1.9 Gy, $p<0.05)$. No correlation was found between contour volume changes and the spinal cord dose increase. Three patients exhibited an increase of 7.0-7.2 Gy to D0.1cc, resulting in a re-plan; these patients were correctly identified using calculations on the CBCT/dCT.

Conclusions: An adaptive re-plan can be triggered using spinal cord doses calculated on the CBCT/dCT. Implementing this trigger can reduce patient appointments and radiation dose by eliminating up to $90 \%$ of additional un-necessary CT scans, reducing the workload for radiographers, physicists, dosimetrists, and clinicians.

Keywords: Adaptive radiotherapy, Radiotherapy, Head and neck, Deformable image registration, Spinal cord, Radiation dose, Image guided radiotherapy

\section{Background}

Intensity Modulated Radiation Therapy (IMRT) and Volumetric Modulated Arc Therapy (VMAT) have become the standard of care for patients receiving radiotherapy for cancer of the head and neck, due to the possibility of sculpting the dose distribution around the PTV and sparing the surrounding healthy tissues and organs at risk (OARs) [1-3]. Reproducible and accurate positioning of

\footnotetext{
* Correspondence: c.mcgarry@qub.ac.uk

${ }^{1}$ Radiotherapy Physics, Northern Ireland Cancer Centre, Belfast City Hospital,

Belfast, Northern Ireland

${ }^{3}$ Centre for Cancer Research and Cell Biology, Queen's University Belfast,

Belfast, Northern Ireland

Full list of author information is available at the end of the article
}

the patient on each treatment fraction is essential for accurate treatment delivery, as even small changes from the reference anatomy of the patient's planning CT scan (pCT) have the potential to compromise the PTV coverage or sparing of healthy tissues. The use of patient immobilisation devices, such as thermoplastic shells, mouth bites, and handgrips, along with image-guided radiotherapy (IGRT) can assist in setting up the patient on each fraction and maintaining their position during treatment delivery.

A head and neck treatment course can last up to 7 weeks and patients commonly experience anatomical changes over the course of their treatment. For example, 
weight loss, changes in tumour or nodal volumes, shifting of fluids and muscle mass, and post-operative changes have been widely reported (see for example [4] and references therein). The potential clinical impact of anatomical changes on the dose distribution for both the PTV and the OARs has been explored in the literature (see for example Brouwer et al [5]). It is possible that the PTV coverage could be compromised [6], the maximum dose to the spinal cord could exceed tolerance, potentially resulting in paralysis, pain, and sensory deficits [7], and that sparing of other OARs could be ineffective, for example causing damage to the parotid glands leading to xerostomia [8]. Modern conformal techniques continue to improve dosimetry and minimise negative clinical sequelae but there remains scope for improvement.

Adaptive radiotherapy (ART) is an attractive solution; the patient's plan can be adjusted to their changing anatomy, rather than assuming that it is identical to the pCT. ART can be either on-line, where any required changes to the plan are made on the same day, or offline, where plan adaptations are performed after treatment and implemented for subsequent fractions. Online ART is technically challenging, requiring accelerated re-planning, including contouring, re-optimisation of fields, and checking, all with the patient in the treatment position in the treatment room [9]. A range of off-line ART methods is reported in the literature for $\mathrm{H} \& \mathrm{~N}$ patients. These include scheduling regular re-scan CTs (rCT) acquired on the CT-Sim in the treatment position, effectively acquiring new pCT scans [10-20], or using in-treatment-room imaging, for example verification MV-CTs [21-24], kV-CBCTs [19, 22, 25-30], and the relatively uncommon use of CT-on-rails [4, 31, 32]. The use of ART for $H \& N$ patients with MR-linacs has also recently been reported [33].

In practice, implementing ART can be time and resource intensive. Carrying out regular, potentially unnecessary, rCTs results in an additional patient appointment and additional patient dose, as well as extra resource and staff time. Using in-room verification imaging for ART exploits an existing step in patient treatment given that most centres now use daily or weekly $\mathrm{CBCT}$ for patient positioning, requiring no additional patient dose or appointments. However, the difficulty of calculating dose on CBCTs has been well-documented [34]. Proposed solutions include density overrides of the CBCT Hounsfield Units (HUs) [35], using CBCT anatomy-specific HU- Relative Electron Density (RED) calibration curves [36, 37], and exploiting deformable image registration (DIR) of the pCT to CBCT [25-27].

It has been reported that only a subset of the head and neck patient populations will benefit from ART $[5,21,38]$, and identifying these patients prior to their radiotherapy treatment is difficult [22]. There is, however, the potential to better allocate the use of resource and staff time by selecting patients who will benefit from ART during treatment based upon a clinical trigger, for example a change in patient size that would impact upon the coverage of the PTV, spinal cord doses, or OAR doses.

Following our protocol, head and neck patients have an $\mathrm{rCT}$ when potentially unacceptable variations in anatomy from the $\mathrm{pCT}$ are observed on the $\mathrm{kV} \mathrm{CBCT}$ used for verification imaging. This may include significant weight loss or changes in flexion or extension of the cervical spine that cannot be adjusted with set up. The patient's original treatment plan is copied to the rCT scan using a rigid registration, using a bony match with further manual adjustments if required, to the $\mathrm{pCT}$, and the dose to the spinal cord is evaluated. If the spinal cord dose is outside tolerance, then a re-plan will be carried out on the rCT. On some occasions, an $\mathrm{rCT}$ and replan will be required for other reasons, such as tumour growth requiring a new immobilisation device; in these cases, the clinical decision to re-plan has already been taken prior to the rCT being acquired. This work seeks to identify a clinical re-plan trigger based on the dose to the spinal cord, resulting in an improvement to the patient experience by reducing the numbers of unnecessary rCTs based on spinal cord dosimetry queries and the associated additional workload for radiographers, clinicians, physicists and dosimetrists.

\section{Methods}

Thirty-five patients treated for cancer of the head and neck that required an $\mathrm{rCT}$ during the course of their treatment were identified for inclusion in this retrospective study. The patients were immobilised for the acquisition of their pCT, consisting of a helical scan with 2.5 $\mathrm{mm}$ slice width, and treatment using a thermoplastic mask. The patients were treated with VMAT (optimisation algorithm PRO 10028 (Varian Medical Systems, Palo Alto)) with one of the following dose prescriptions: 70.0 Gy in 35 fractions, 66.0 Gy in 33 fractions, or 60.0 Gy 30 fractions. Four patients were excluded from the study as their rCT was carried out prior to their first treatment fraction. The images analysed for each of the remaining 31 patients consisted of the $\mathrm{pCT}$, the $\mathrm{CBCT}$ just prior to the acquisition of the $\mathrm{rCT}$, and the $\mathrm{rCT}$.

The volume of the body contour for each patient was evaluated on the $\mathrm{pCT}, \mathrm{CBCT}$, and $\mathrm{rCT}$, to investigate potential correlation between the volume of the body contour, as a surrogate for anatomical changes, and spinal cord dose changes. ROIs were carefully created on each scan and the contours limited to within the ROIs, using the Contouring workspace of Varian Eclipse, so that the same scan length was compared. 
Rigid registrations between the $\mathrm{pCT}$ and $\mathrm{rCT}$, and $\mathrm{pCT}$ and $\mathrm{CBCT}$, were created using the Image Registration module of Eclipse, or reviewed if already present, to enable copying of plans and contours. Using the registration, the original treatment plan with the full treatment prescription was copied to the $\mathrm{CBCT}$ and $\mathrm{rCT}$. The spinal cord contours were created on the CBCTs by a single observer, by copying the original $\mathrm{pCT}$ spinal cord contour and adjusting the contour on each slice to account for any changes in spinal cord position. The spinal cord contours on the CBCT were verified by a clinician for a selection of the patients in the study. The dose for the full dose prescription of the treatment plans (to facilitate comparison between the cohort of patients) was re-calculated on the new images (CBCT and $\mathrm{rCT}$ ) using Varian Eclipse AAA v13.6.23 (Varian Medical Systems). For the calculations on the CBCT, the standard CT HURED conversion curve was applied with no further density overrides. The D0.1cc to spinal cord was evaluated using the DVH for the doses from the CBCT and rCT.

The $\mathrm{pCT}$ was then deformed to the CBCT, creating a DIR, using the SmartAdapt ${ }^{\circ}$ workspace of Varian Eclipse to form the deformed CT (dCT). The default deformable registration algorithm in SmartAdapt ${ }^{\circ}$ is a modified demons algorithm [39]. The ROI for the DIR was carefully selected to obtain a satisfactory registration that covered the full extent of the high dose PTV, where possible. The DIRs were evaluated in line with the recommendations of TG132 [40]. The deformation vector field (DVF) created from the DIR was checked for non-physical results such as folding by viewing the deformation grid, which is deformed according to the DVF, and the deformation distance colour map showing the length of the deformation vectors. The DVF also warps the $\mathrm{pCT}$ contours; these were visually inspected for inconsistencies but not found to require adjustments. The dose was calculated on the $\mathrm{dCT}$ and the maximum dose to $0.1 \mathrm{cc}$ of the spinal cord (D0.1cc) evaluated from the DVH.

The pCT spinal cord D0.1cc was compared to that calculated on the $\mathrm{rCT}$, to evaluate whether these patients had an increase in spinal cord doses. The spinal cord D0.1cc calculated on the CBCT and $\mathrm{dCT}$ was compared to that calculated on the $\mathrm{rCT}$, considered the gold standard, in order to identify the possibility of using these methods as a re-plan trigger. The spinal cord tolerance used for planning is D0.1 cc $<45.0 \mathrm{~Gy}$.

Microsoft Excel and SPSS Statistics (IBM) were used to perform the statistical analysis, with paired sample tests used to examine the statistical significance of changes in the body contour and spinal cord. Where the distribution of the data was found to be normal on examination of histograms, q-q plots, and the results of a Kolmogorov-Smirnoff test, the student's paired sample t-test was used. Where normality could not be assumed, a Wilcoxin signed rank test was carried out. Statistical significance was assumed with $p<0.05$.

\section{Results \\ Volume metrics}

All patients experienced a statistically significant change in the volume of the body contour between the $\mathrm{pCT}$ and rCT. For the majority of patients (25/31), the body contour decreased in volume (average $5.2 \pm 5.5 \%$, paired sample $\mathrm{t}$-test $\mathrm{p}<0.05)$ suggesting that the patients lost weight in this time. There is a weak correlation $\left(R^{2}=\right.$ $0.49)$ between fraction of re-scan and change in contour volume, with patients re-scanned later in their course of treatment exhibiting a larger reduction in contour volume (Fig. 1). Of the remaining six patients, one had almost no change in contour volume. The other five patients had an increase in body contour volume (average $4.4 \pm 4.0 \%$, paired sample t-test $p<0.05$ ); for three patients this increase appears to be due to an increase in tumour volume. No correlation was found in this group of patients between change in volume of the body contour and change in spinal cord dose.

\section{Spinal cord doses: $\mathrm{pCT}$ to $\mathrm{rCT}$}

Currently, patients in our centre will receive an ART replan according to the spinal cord dose on the rCT. The majority of the patients in this study experienced an increase in D0.1cc to the spinal cord from pCT to rCT, with the mean treatment fraction for the $\mathrm{rCT}$ being fraction 17 (range 1-30, standard deviation 9). The median spinal cord D0.1cc on the pCT was $40.3 \mathrm{~Gy}$ (interquartile range $3.6 \mathrm{~Gy})$. The median dose increase to D0.1cc was $0.7 \mathrm{~Gy}$ (inter-quartile range $0.2-1.9 \mathrm{~Gy}, p<0.05 \mathrm{Wil}-$ coxin signed rank test). This data is shown in Fig. 2a as a frequency histogram. The majority of the patients have a relatively small change in spinal cord D0.1cc of \pm 2.5

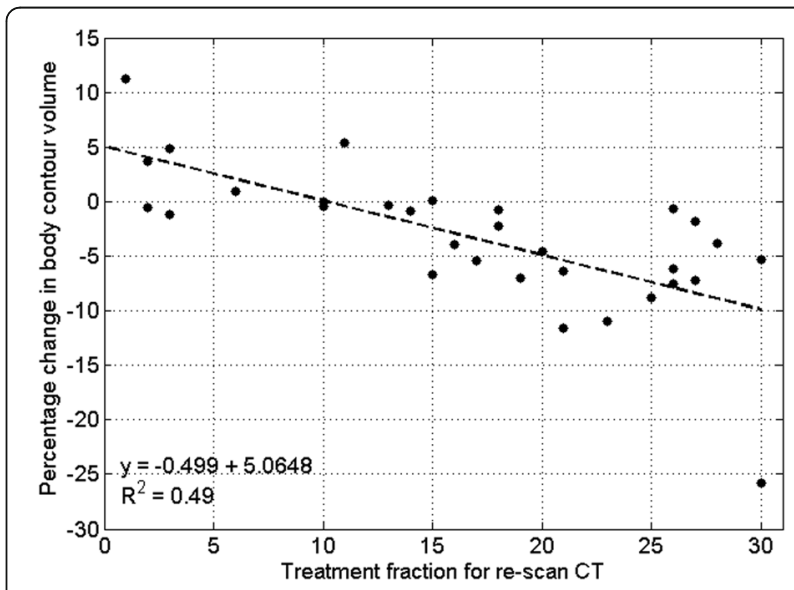

Fig. 1 The percentage change in body contour volume between the $\mathrm{PCT}$ and $\mathrm{rCT}$ with the fraction of treatment for re-scan. There is a larger reduction in body contour volume as treatment progresses 

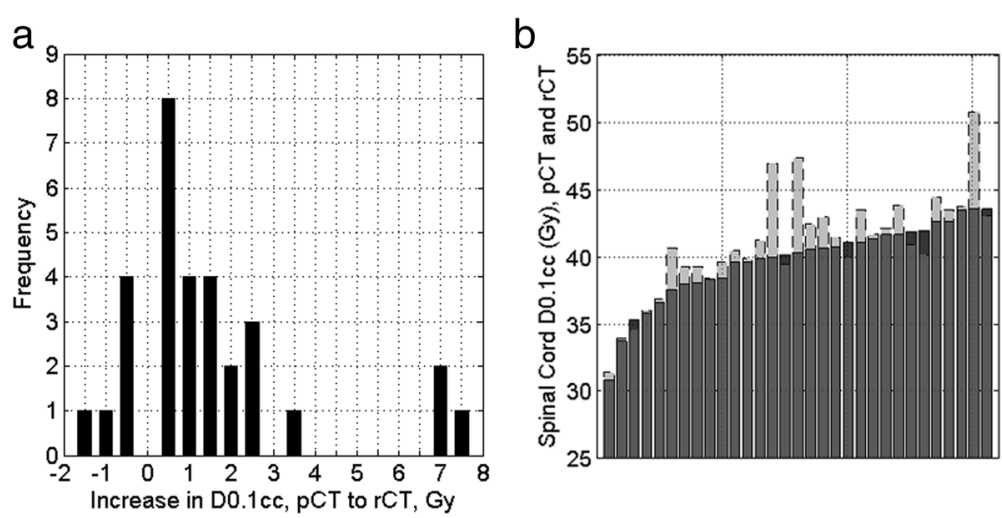

Fig. 2 Spinal cord dose increases for the patients in this study. a shows the frequency distribution of the differences in spinal cord D0.1cc doses between pCT and rCT. The majority of patients experience a small change in spinal cord dose. The distribution is skewed by a small number of patients showing a large increase in D0.1cc. $\mathbf{b}$ shows the changing doses for each patient between the pCT and the $r C T$. The pCT dose is in grey with a solid outline and the $\mathrm{rCT}$ dose shown as a stacked light grey bar with a dashed outline. For the patients whose D0.1cc to spinal cord was reduced at the $\mathrm{rCT}$ with respect to the $\mathrm{pCT}$, the decrease is shown as a dark outline over the initial $\mathrm{pCT}$ dose

Gy. The distribution is skewed by three patients who have spinal cord D0.1cc increases of $\geq 7.0 \mathrm{~Gy}$; the same three patients exceeded the tolerance to D0.1cc of spinal cord of $45.0 \mathrm{~Gy}$. Figure 2b shows the data presented as increases for each patient from the $\mathrm{pCT}$ dose.

\section{Spinal cord doses: calculating on the CBCT}

Figure $3 \mathrm{a}$ shows the results of calculating on the CBCT to estimate spinal cord D0.1cc, with the difference between calculating on the CBCT and the $\mathrm{rCT}$ plotted against the dose calculated on the $\mathrm{rCT}$ as the gold standard. All values calculated using the CBCT values agree with the $\mathrm{rCT}$ to within $\pm 3.4 \mathrm{~Gy}$.

\section{Spinal cord doses: calculating on the dCT}

Figure $3 \mathrm{~b}$ shows the results of calculating on the $\mathrm{dCT}$ to estimate spinal cord D0.1cc. The mean difference from the $\mathrm{rCT}$ is $0.2 \mathrm{~Gy}$. All values calculated using the $\mathrm{dCT}$ agree with the $\mathrm{rCT}$ within $\pm 3.4 \mathrm{~Gy}$.

It appears from Fig. $3 a$ and $b$ that there could be a relationship between the magnitude of the change in spinal cord dose and the spinal cord dose on the rCT. Upon analysis, no correlation $\left(\mathrm{R}^{2}<0.2\right)$ was found between the absolute change in spinal cord dose and the rCT spinal cord dose.

Although there is an uncertainty of \pm 3.4 Gy in determining the D0.1cc using the $\mathrm{dCT}$ or $\mathrm{CBCT}$, both methods correctly identified three patients whose D0.1cC to the spinal cord exceeded the $45.0 \mathrm{~Gy}$ spinal cord tolerance. These same three patients experienced large increases in spinal cord D0.1cc, as seen in Fig. 2.

Adopting a trigger level of $\mathrm{D} 0.1 \mathrm{cC}=45.0 \mathrm{~Gy}$ to the spinal cord based on the $\mathrm{dCT}$ calculations resulted in no false positives or negatives, and one false positive using
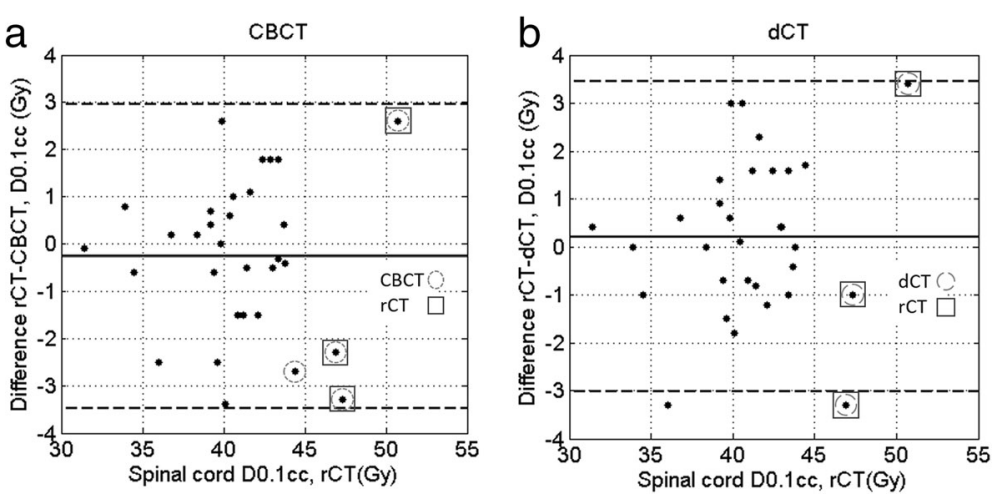

Fig. 3 Bland-Altmann charts illustrating the difference between the D0.1cc to spinal cord for each patient as calculated on (a) the CBCT and (b) the dCT, compared to the gold standard of calculating on the rCT. The solid line indicates the mean difference and the dashed lines the $95 \%$ confidence intervals. On each panel, the data points representing patients triggered for a re-plan using the CBCT and dCT have been highlighted with a circle and compared to those triggered on the $\mathrm{rCT}$, which are highlighted with a square 
the $\mathrm{CBCT}$ calculations. Considering the uncertainty in the dose calculation, a conservative action level on the $\mathrm{dCT} / \mathrm{CBCT}$ of D0.1cc $=42.0$ Gy could be adopted. With this criteria, 20/28 patients would have been spared an $\mathrm{rCT}$ using the $\mathrm{dCT}$, and $17 / 28$ patients when using the CBCT.

\section{Discussion}

For a group of 31 head and neck patients, patient size, as represented by the volume of the patient's body contour, cannot be considered as a useful trigger for ART based on spinal cord doses. However, calculating on the patient's verification $\mathrm{CBCT}$ images, or on the $\mathrm{pCT}$ deformed to the CBCT, can be used to provide an estimate of spinal cord doses and thus trigger an adaptive re-plan where necessary. This will reduce the number of unnecessary additional CT scans, especially considering that the number of patients requiring an ART plan due to spinal cord doses is small.

It has been proposed that weight loss in head and neck patients influences patient shape (e.g. $[4,16])$ and positioning $[11,41]$, and therefore has the possibility of affecting the dose distribution with potential increases to OARs (for example [5]). In particular, weight loss has been correlated to an increase in the maximum dose to the spinal cord [42] and doses to the parotid glands [23, 42]. A number of authors implement ART in H\&N patients according to a change in body contours; for example a $5 \mathrm{~mm}$ margin around the target volume [25] or $1 \mathrm{~cm}$ change anywhere in the body contour $[22,43]$.

Whilst we observed statistically significant changes in the body contour over time, suggesting weight loss in line with other published data [21, 44], there was no significant correlation found between change in contour volume and change in dose to the spinal cord. This is in agreement with results published by Noble et al, who found no correlation between shape change and change in spinal cord doses [21]. This suggests that change in patient shape cannot be used as a clinically useful replan trigger according to spinal cord dose. Similarly, in this group of patients some re-plans were carried out for other reasons, including disease progression and requirement of a new immobilisation mould. A larger change in body contour volume was not found to correlate with re-plan requirement for any reason. This finding is in agreement with Hansen et al [44], who observed no statistically significant correlations in any of their studied metrics between patients re-planned due to weight loss and those re-planned for other reasons.

In this group of patients, there was a small but statistically significant change in D0.1cc to the spinal cord of $0.7 \pm 2.2 \mathrm{~Gy}$, in agreement with values in the literature where average increases in $D_{\max }$ were reported in the range $1.1-1.4 \mathrm{~Gy}[14,21,28,45] .81 \%$ of the patients had an increase in dose to cord; this is comparable to values reported in the literature, for example Wang et al [42] reported $87 \%$ and Hansen et al. [44] $100 \%$ of patients showing an increase in dose to the cord.

For three patients there was an increase in spinal cord dose between the pCT and rCT of 7.0-7.2 Gy, resulting in doses exceeding the cord tolerance for the plan. Only one of these three patients had an initial spinal cord dose close to the planning tolerance, at $43.5 \mathrm{~Gy}$. This has also been reported in the literature, with between 5 and $30 \%$ of patient cohorts exhibiting increases that push the spinal cord dose out of tolerance [30, 46-48]. On review of the CT and CBCT imaging for these three patients, the large dose increases can be attributed to significant positioning changes due to curvature of the spine, pushing the spinal cord into a high dose region.

Using the CBCT and $\mathrm{dCT}$ to calculate D0.1cc to spinal cord and trigger a re-plan is feasible for these patients. The three patients with large spinal cord dose increases, resulting in tolerance being exceeded, were correctly identified using both methods. Variations in calculated doses on the CBCT with respect to CT are $\pm 3.4 \mathrm{~Gy}$, in line with Fotina et al [35], who reported a 5\% variation in target coverage on the CBCT, and Naufal et al [49] who found a $7 \%$ variation in H\&N CBCT calculations.

The method presented here involves recalculating the entire dose prescription on the rCT. Calculating instead a cumulative spinal cord D0.1cc from the $\mathrm{pCT}$ and $\mathrm{rCT}$ results in one patient exceeding the D0.1cc tolerance of 45.0 Gy with an increase of 6.8 Gy. Despite not exceeding the planning tolerance, the remaining two of the three patients previously identified also exhibited large increases in spinal cord doses of 3.4 and $3.6 \mathrm{~Gy}$, well above the median cumulative increase of $0.4 \mathrm{~Gy}$. A cumulative analysis assumes that the patient's spinal cord dose will remain stable for the remainder of their treatment. Calculating the entire prescription therefore presents a conservative method by which to trigger a replan for the most at-risk patients, regardless of the time point in the patient's treatment course.

In this study, there were only three patients with spinal cord doses above the tolerance of D0.1 cc of $45 \mathrm{~Gy}$ against which to test our re-plan trigger. This data, however, was gathered over a period of 2.5 years with on average $17 \mathrm{H} \& \mathrm{~N}$ VMAT plans created per month; this supports the conclusion that selectivity for ART in this cohort of patients is of utmost importance to achieve the best balance of additional workload to clinical benefit. This will result in a potential reduction from 9 to $1 \%$ of $\mathrm{H} \& \mathrm{~N}$ patients requiring a $\mathrm{rCT}$, reducing the need for additional dose to these patients. Furthermore, we have only examined the dose to the spinal cord. This approach was taken as our clinical decision-making is based upon 
spinal cord doses on the rCT. Other publications have reported in depth the positional and dosimetric changes to other OARs, with the parotid glands the OAR of focus. Dosimetric sparing of the parotids has been reported to be approximately 1 Gy to mean parotid dose [12, 19, 32], although one study reported increases to the parotid mean dose of, on average, 4 Gy to $60 \%$ of the patient cohort [50]. The clinical benefit of such sparing with re-planning has been questioned [19], with the NTCP increase for grade 3 xerostomia estimated at $0.03 \%$ in one study [12]. There is again evidence that these dosimetric increases push the OAR dose above tolerance in only a small minority of cases [47]. Since the conclusion of this study, our centre has moved to daily CBCT imaging for $\mathrm{H} \& \mathrm{~N}$ patients. With more frequent imaging and therefore an improving evidence base, other lower priority OARs that may benefit from ART, for example the parotid glands, could also be evaluated using our method.

\section{Conclusions}

For a group of $31 \mathrm{H} \& \mathrm{~N}$ patients, change in body contour volume was not found to indicate the necessity of a replan according to spinal cord dose tolerances. However, significant positional changes such as neck flexion and extension can. The spinal cord doses as calculated on the patients' verification CBCT image or the $\mathrm{pCT}$ deformed to the CBCT anatomy can be used as a trigger to identify the subset of patients that require an adaptive re-plan, reducing the need to acquire unnecessary additional CT scans. The number of patients that require a re-plan due to spinal cord doses is small, indicating that ART for spinal cord doses is necessary only for a minority of head and neck patients, despite the large anatomical changes that they experience.

\section{Abbreviations}

ART: Adaptive radiotherapy; CBCT: Cone beam CT; dCT: Deformed CT; DIR: Deformable image registration; DVF: Deformation vector field; DVH: Dose-volume histogram; H\&N: Head and neck; HU: Hounsfield units; OAR: Organ at risk; $\mathrm{pCT}$ : Planning CT; PTV: Planning target volume; rCT: Rescan CT; RED: Relative electron density

\section{Acknowledgments}

Work was performed using treatment planning workstations purchased through charitable funds provided by Friends of the Cancer Centre (registered with The Charity Commission for Northern Ireland NIC101345).

\section{Authors' contributions}

Conception and design: LB, CA, DI, CMcG. Contour verification: KR. Collection and assembly of data, analysis and interpretation: LB. Manuscript writing: LB, $C A, D I, C M c G$. Final approval of manuscript: All authors read and approved the final manuscript

\section{Funding}

None to declare.

\section{Availability of data and materials}

The dataset supporting the conclusions of this article is included within the article. Additional data are available from the corresponding author on reasonable request.

Ethics approval and consent to participate

Not applicable.

\section{Consent for publication}

Not applicable.

\section{Competing interests}

The authors declare that they have no competing interests.

\section{Author details}

${ }^{1}$ Radiotherapy Physics, Northern Ireland Cancer Centre, Belfast City Hospital, Belfast, Northern Ireland. ${ }^{2}$ Clinical Oncology, Northern Ireland Cancer Centre, Belfast City Hospital, Belfast, Northern Ireland. ${ }^{3}$ Centre for Cancer Research and Cell Biology, Queen's University Belfast, Belfast, Northern Ireland.

Received: 4 July 2019 Accepted: 16 October 2019

Published online: 01 November 2019

\section{References}

1. Nutting CM, Morden JP, Harrington KJ, Urbano TG, Bhide SA, Clark C, Miles EA, Miah A, Newbold K, Tanay M, Adab F, Jefferies SJ, Scrase C, Yap BK, A'Hern RP, Sydenham MA, Emson M, Hall E. Parotid-sparing intensity modulated versus conventional radiotherapy in head and neck cancer (PARSPORT): a phase 3 multicentre randomised controlled trial. Lancet Oncol. 2011;12(2):127-36.

2. Infusino E. Clinical utility of RapidArcTM radiotherapy technology. Cancer Manag Res. 2015;7:345-56.

3. Osborn J. Is VMAT beneficial for patients undergoing radiotherapy to the head and neck? Radiography. 2017;23(1):73-6.

4. Barker J, Garden A, Ang KK, O'Daniel J, Wang H, Court L, Morrison W, Rosenthal D, Chao KC, Tucker S, Mohan R, Dong L. Quantification of volumetric and geometric changes occurring during fractionated radiotherapy for head and neck cancer using an integrated CT/linear accelerator system. Int J Radiation Oncology Biol Phys. 2004;59:960-70.

5. Brouwer C, Steenbakkers R, Langendijk J, Sijtsema N. Idenitfying patients who may benefit from adaptive radiotherapy: does the literature on anatomic and dosimetric changes in head and neck organs at risk during radiotherapy provide information to help? Radiother Oncol. 2015;115:285-94.

6. Bhide SA, Davies M, Burke K, McNair HA, Hansen V, Barbachano Y, El-Hariry IA, Newbold K, Harrington KJ, Nutting CM. Weekly volume and dosimetric changes during chemoradiotherapy with intensity-modulted radiation therapy for head and neck cancer: a prospective observational study. Int J Radiation Oncology Biol Phy. 2010;76(5):1360-8.

7. Kirkpatrick JP, Van Der Kogel AJ, Schultheiss TE. Radiation dose-volume effects in the spinal cord. Int J Radiat Oncol Biol Physics. 2010;76(3):S42-9.

8. Pinna R. Xerostomia induced by radiotherapy: an overview of the physiopathology, clinical evidence, and management of the oral damage. Ther Clin Risk Manag. 2015;4:171-88.

9. Lim-Reinders S, Keller BM, AI-Ward S, Sahgal A, Kim A. Online adaptive radiation therapy. Int J Radiation Oncol Biol Phys. 2017;99(4):994-1003.

10. Robar JL, Day A, Clancey J, Kelly R, Yewondwossen M, Hollenhorst H, Rajaraman M, Wilke D. Spatial and dosimetric variability of organs at risk in head-and-neck intensity-modulated radiotherapy. Int J Radiation Oncology Biol Phys. 2007:68:1121-30.

11. O'Daniel JC, Garden AS, Schwartz DL, Wang H, Ang KK, Ahamad A, Rosenthal DI, Morrison WH, Asper JA, Zhang L, Tung S-M, Mohan R, Dong L. Parotid gland dose in intensity-modulated radiotherapy: is what you plan what you get? Int J Radiation Oncol Biol Phys. 2007;69:1290-6.

12. Marzi S, Pinnaro P, D'Alessio D, Strigari L, Bruzzaniti V, Giordano C, Giovinazzo G, Marucci L. Anatomical and dose changes of gross tumour volume and parotid glands for head and neck cancer patients during Intenisty-modulated radiotherapy: effect on the probability of xerostomia incidence. Clin Oncol. 2012;53:e54-62. 
13. Kataria T, Gupta D, Goyal S, Basu T, Abhishek A, Narang K, Banerjee S, Nasreen S, Sambasivam S, Dhyani A. Clinical outcomes of adaptive radiotherapy in head and neck cancers. Br J Radiol. 2016;88:20160085.

14. Ahn PH, Chen CC, Ahn AL, Hong L, Scripes P, Shen J, Lee CC, Miller E, Kalnicki S, Garg MK. Adaptive planning in intensity-modulated radiation therapy for head and neck cancers: single institution experience and clinical implications. Int J Radiat Oncol Biol Phys. 2011;80:677-85.

15. Capelle L, Mackenzie M, Field C, M. Parliament, Ghosh S, Scrimger R. Adaptive radiotherapy uisng helical tomotherapy for head and neck cancer in definitive and postoperative settings: initial results. Clin Oncol. 2012;24:208-15.

16. Beltran M, Ramos M, Rovira JJ, Perez-Hoyos S, Sancho M, Puertas E, Benavente S, Ginjaume M, Giralt J. Dose variations in tumour volumes and organs at risk during IMRT for head and neck cancer. J Appl Clin Med Phys. 2012;13:101-11.

17. Wu Q, Chi Y, Chen P, Krauss D, Yan D, Martinez A. Adaptive replanning strategies accounting for shrinkage in head and neck IMRT. Int J Radiat Oncol Biol Phys. 2009;75:924-32.

18. Surucu M, Shah KK, Roeske JC, Choi M, Small W Jr, Emami B. Adaptive radiotherapy for head and neck cancer: implications for clinical outcomes. Technol Cancer Res Treat. 2017;16(2):218-23.

19. Hvid CA, Elstrom UV, Jensen K, Grau C. Cone-beam computed tomography (CBCT) for adative image guided head and neck radiation therapy. Acta Oncol. 2018;57(4):552-6.

20. Huang $H$, Lu H, Feng G, Jiang H, Chen J, Cheng J, Pang Q, Lu Z, Gu J, Peng L, Deng S, Mo Y, Wu D, Wei Y. Determining appropriate timing of adaptive radiation therapy for nasopharyngeal carcinoma during intensity-modulated radiation therapy. Radiat Oncol. 2015;10:192.

21. Noble DJ, Yeap P-L, Seah SY, Harrison K, Shelley LE, Romanchikova M, Bates AM, Zheng Y, Barnett GC, Benson RJ, Jefferies SJ, Thomas SJ, Jena R, Burnet NG. Anatomical change during radiotherapy for head and neck cancer, and its effect on delivered dose to the spinal cord. Radiother Oncol. 2019;130:32-8.

22. Brown E, Owen R, Harden F, Mengersen K, Oestreich K, Houghton W, Poulsen M, Harris S, Lin C, Porceddu S. Head and neck adaptive radiotherapy: predicting the time to re-plan. Asia-Pac J Clin Oncol. 2016;12:460-7.

23. Lee C, Langen KM, Lu W, Haimerl J, Schnarr E, Ruchala KJ, Olivera GH, Meeks SL, Kupelian PA, Shellenberger TD, Manon RR. Assessment of parotid gland dose changes during head and neck cancer radiotherapy using daily megavoltage computed tomography and deformable image registraion. Int J Radiat Oncol Biol Phys. 2008;71:1563-71.

24. Han C, Chien Y-J, Liu A, Schultheiss TE, Wong JYC. Actual dose variation of parotid glands and spinal cord for nasopharyngeal patients during radiotherapy. Int J Radiat Oncol Biol Phys. 2008;70:1256-62.

25. Veiga C, McClelland J, Moinuddin S, Lourenco A, Ricketts K, Annkah J, Modat M, Ourselin S, D'Souza D, Royle G. Toward adaptive radiotherapy for head and neck patients: feasibility study in using CT-to-CBCT deformable registration for "dose of the day" calculations. Med Phys. 2014;41:031703.

26. Elstrom U, Wysocka B, Muren L, Petersen J, Grau C. Daily kV cone-beam CT and deformable image registration as a method for studying dosimetric consequences of anatomic changes in adaptive IMRT of head and neck cancer. Acta Oncol. 2010;49:1101-8.

27. Jin X, Hu W, Shang H, Han C, Yi J, Zhou Y, Xie C. CBCT-based volumetric and dosimetric evaluation of volumetric-modulated arc radiotherapy in the treatment of nasopharyngeal cancer patients. Radiat Oncol. 2013;8:279.

28. Ho KF, Marchant T, Moore C, Webster G, Rowbottom C, Penington H, Lee L, Yap B, Sykes A, Slevin N. Monitoring dosimetric impact of weight loss with kilovoltage $(\mathrm{kV})$ cone beam CT (CBCT) during parotid-sparing IMRT and concurrent chemotherapy. Int J Radiation Oncol Biol Phys. 2012;82(3):e375-82.

29. Chen S, Le Q, Mutaf Y, Lu W, Nichols EM, Yi BY, Leven T, Prado KL, D'Souza WD. Feasibility of CBCT-based dose with a patient-specific stepwise HU-todensity curve to determine time of replanning. J Appl Clin Med Phys. 2017; 18(5):64-9.

30. Vickress JR, Battista J, Barnett R, Yartsev S. Online daily assessment of dose change in head and neck radiotherapy without dose-recalculation. J Appl Clin Med Phys. 2018;19(5):659-65.

31. Schwartz DL, Garden AS, Thomas J, Chen Y, Zhang Y, Lewin J, Chambers MS, Dong $L$. Adaptive radiotherapy for head and neck cancer: initial clinical outcomes from a prospective trial. Int J Radiat Oncol Biol Phys. 2012;83:986-93.

32. Schwartz DL, Garden AS, Shah SJ, Chronowksi G, Sejpal S, Rosenthal DI, Chen Y, Zhang Y, Zhang Y, Zhang L, Wong PF, Garcia JA, Ang KK, Dong L. Adaptive radiotherapy for head and neck cancer - Dosimetric results from a prospective clinical trial. Radiother Oncol. 2013;106:80-4.
33. Chuter RW, Pollitt A, Whitehurst P, Mackay RI, Van Herk M, McWilliam A. Assessing MR-linac radiotherapy robustness for anatomical changes in head and neck cancer. Phys Med Biol. 2018;63:125020.

34. Giacometti V, King RB, Agnew CE, Irvine DM, Jain S, Hounsell AR, McGarry CK. An evaluation of techniques for dose calculation on cone beam computed tomography. Br J Radiol. 2019;92(1096):20180383.

35. Fotina I, Hopfgartner J, Stock M, Steininger T, Lutgendorf-Caucig C, Georg D. Feasibility of CBCT-based dose calculation: comparative analysis of HU adjustment techniques. Radiother Oncol. 2012;104:249-56.

36. Richter A, Hu Q, Steglich D, Baier K, Wilbert J, Guckenberger M, Flentje M. Investigation of the usability of conebeam CT data sets for dose calculation. Radiat Oncol. 2008;3:42.

37. Rong Y, Smilowitz J, Tewatia D, Tome WA, Paliwal B. Dose calculations on kV cone beam CT images: an investigation of the HU-denisty converison stability and dose accuracy using the site-specific calibration. Med Dosim. 2010;35:195-207.

38. Gregoire V, Langendijk JA, Nuyts S. Advances in radiotherapy for head and neck cancer. J Clin Oncol. 2015;33(29):3277-84.

39. Wang H, Dong L, O'Daniel J, Mohan R, Garden AS, Kian Ang K, Kuban DA, Bonnen M, Chang JY, Cheung R. Validation of an accelerated demons algorithm for deformable image registration in radiation therapy. Phys Med Biol. 2005;50(12):2887-905.

40. Brock KK, Mutic S, McNutt TR, Li H, Kessler ML. Use of image registration and fusion algorithms and techniques in radiotherapy: report of the AAPM radiation therapy committee task group no. 132. Med Phys. 2017;44(7):e43-76.

41. Ayyalusamy A, Vellaiyan S, Shanmugam S, Illamurugu A, Gandhi A, Shanmugam T, Murugesan K. Feasability of offline Head \& Neck adaptive radiotherapy using deformed planning $C T$ electron denisty mapping on weekly cone beam computed tomography. Br J Radiol. 2017;90:20160420.

42. Wang X, Lu J, Xiong X, Zhu G, Ying H, He S, Hu S, Hu C. Anatomic and dosimetric changes during the treatment course of intensity-modulated radiotherapy for locally advanced nasopharyngeal carcinoma. Med Dosim. 2010;35(2):151-7.

43. Duma MN, Kampfer S, Schuster T, Winkler C, Geinitz H. Adaptive radiotherapy for soft tissue changes during helical tomotherapy for head and neck cancer. Strahlenther Onkol. 2012;188:243-7.

44. Hansen E, Bucci MK, Quivey JM, Weinberg V, Xia P. Repeat CT imaging and replanning during the course of IMRT for head and neck cancer. Int J Radiat Oncol Biol Phys. 2006;64:355-62.

45. Cozzolino M, Fiorentino A, Oliviero C, Pedicini P, Clemente S, Califano G, Caivano R, Chiumento C, Fusco V. Volumetric and dosimetric assessment by cone-beam computed tomography scans in head and neck radiation therapy: a monitoring in four phases of treatment. Technol Cancer Res Treat. 2014;13(4):325-35.

46. Fung WWK, Cheung WW, Teo PML. Dosimetric evaluation of a three-phase adaptive radiotherapy for nasopharyngeal carcinoma using helical tomotherapy. Med Dosim. 2012;37:92-7.

47. Graff P, Hu W, Yom SS, Pouliot J. Does IGRT ensure target dose coverage of head and neck IMRT patients? Radiother Oncol. 2012;104:83-90.

48. Cheng H, Wu V, Ngan R, Tang K, Chan C, Wong K, Au S, Kwong D. A prospective study on volumetric and dosimetric changes during intensitymodulated radiotherapy for nasopharyngeal carcinoma patients. Radiother Oncol. 2012;104:317-23.

49. Naufal MP, Abdullah KK, Niyas PN, Sankaran TS, Sasindaran PR. Analysis of dosimetric impacts of cone beam computed tomography-based volumetric modulated arc therapy planning. J Med Imaging Radiat Sci. 2016;47:160-70.

50. Castelli J, Simon A, Louvel G, Henry O, Chajon E, Nassef M, Haigron P, Cazoulat G, Ospina Arango J, Jegoux F, Benezery K, de Crevoisier R. Impact of head and neck cancer adaptive radiotherapy to spare the parotid glands and decrease the risk of xerostomia. Radiat Oncol. 2015;10(6). https://doi. org/10.1186/s13014-014-0318-z.

\section{Publisher's Note}

Springer Nature remains neutral with regard to jurisdictional claims in published maps and institutional affiliations. 